\title{
Uma Breve Análise Bibliométrica Sobre Biblioteca no Meio Digital
}

\author{
A Bibliometric Analysis on Digital Library
}

Ananda Mayara Batista Rocha ${ }^{a}$; Fátima Aparecida da Silva Dias*a

\begin{abstract}
${ }^{a}$ Unopar, Programa de Pós-Graduação Stricto Sensu em Metodologias para o Ensino de Linguagens e suas Tecnologias. PR, Brasil. *E-mail: fatimadias.consultoria@gmail.com
\end{abstract}

\begin{abstract}
Resumo
O objetivo deste artigo é apresentar a análise sobre o tratamento que a comunidade científica da Ciência da Informação dá ao tema Bibliotecas no ambiente digital. Para isso se utilizou das leis bibliométricas para as análises métricas: Lei de Bradford, Lei de Lotka, Lei de Zipf. Tratase de uma pesquisa descritiva, teórico-conceitual, com lógica dedutiva, quanto aos procedimentos metodológicos foram utilizados dados primários coletados durante as pesquisas e a abordagem do problema é quali-quantitativa. A análise resultou em um conjunto de dados que foram organizados por meio de técnicas de bibliometria dispostos em quadros e gráficos. Constatou-se que a Revista Ciência da Informação foi a que teve a maior frequência de publicação e que a maioria dos artigos possui autoria múltipla, sendo que os autores preferem usar a expressão "biblioteca digital" apenas no título e no resumo.
\end{abstract}

Palavras-chave: Bibliometria. Biblioteca Virtual. Biblioteca Eletrônica. Biblioteca Digital. Biblioteca Online.

\section{Abstract}

The purpose of this article is to analyze the treatment given by the scientific community of Information Science to the theme libraries in the digital environment Thus, bibliometric laws were used for the metric analysis: Bradford's Law, Lotka's Law, Zipf's Law. This is a descriptive, theoretical-conceptual research, with deductive logic, as the methodological procedures used primary data collected during the research and the problem approach is qualitative and quantitative. Data analysis resulted in a set of data that was organized using bibliometric techniques and arranged in charts and graphs. It was found that the journal Ciência da Informação was the one with the highest frequency of publication and that most articles have multiple authorship and the authors prefer to use the expression "digital library" only in the title and abstract.

Keywords: Bibliometrics. Virtual Library. Electronic Library. Digital Library. Online Library

\section{Introdução}

Este estudo apresenta uma breve análise bibliométrica de artigos que possuem no título ou no resumo o termo "biblioteca virtual", "biblioteca eletrônica", "biblioteca digital", "biblioteca on-line" ou "biblioteca online". Justificase este estudo pela necessidade de analisar o tratamento que a comunidade científica da Ciência da Informação dá a esses temas específicos da área. A análise foi realizada com o objetivo de identificar as características da comunidade que publica sobre o tema e obter dados, que respondam à pergunta: com que frequência a comunidade científica escreve sobre biblioteca virtual, biblioteca eletrônica, biblioteca digital, biblioteca on-line (online) nas revistas brasileiras da área de Ciência da Informação? E qual termo é mais usado para publicar sobre o mesmo tema nas revistas brasileiras da área de Ciência e Informação.

\section{Desenvolvimento}

\subsection{Metodologia}

1Foram coletados os seguintes dados: ano da publicação, ISSN para representarem os periódicos, título e subtítulo dos artigos, autor (es), a pesquisa foi realizada pelos descritores: biblioteca virtual, biblioteca eletrônica, biblioteca digital, "biblioteca on-line (online)". Não foi especificada a data durante as pesquisas, entretanto, optou-se por organizar as datas de publicação dos resultados encontrados.

Quadro 1 - Periódicos de Biblioteconomia da base ABCDM

\begin{tabular}{|c|l|}
\hline $\mathbf{N}^{\mathbf{0}}$ & Nome da Revista \\
\hline 1 & Biblos: Revista do Departamento de Biblioteconomia e História (BDBH): 0102-4388; \\
\hline 2 & Brazilian Journal of Information Science: $1981-1640 ;$ \\
\hline 3 & Cadernos de Biblioteconomia (CB): 0102-6607; \\
\hline 4 & Cadernos de Biblioteconomia, Arquivística e Documentação: 0007-9421; \\
\hline 5 & Ciência da Informação: 0100-1965 e 1518-8353; \\
\hline 6 & DatagramaZero: $1517-3801 ;$ \\
\hline
\end{tabular}




\begin{tabular}{|c|l|}
\hline 7 & Em Questão: Revista da Faculdade de Biblioteconomia e Documentação da UFRGS: 1807-8893 e 1808-5245; \\
\hline 8 & Encontros Bibli: Revista Eletrônica de Biblioteconomia e Ciência da Informação: 1518-2924; \\
\hline 9 & ENANCIB, Encontro Nacional de Pesquisa em Ciência da Informação - ENANCIB: 2177-3688 \\
\hline 10 & InCID: Revista de Ciência da Informação e Documentação (INCID): 2178-2075 \\
\hline 11 & Informação \& Informação (II): 1414-2139 e 1981-8920; \\
\hline 12 & Informação \& Sociedade (ISE): Estudos: 0104-0146 e 1809-4783; \\
\hline 13 & Informare: Cadernos do Programa de Pós-Graduação em Ciência da Informação: 0104-9461; \\
\hline 14 & Perspectivas em CI (PCI): 1413-9936 e 1981-5344; \\
\hline 15 & Perspectivas em Gestão \& Conhecimento (PGC): 2236-417X; \\
\hline 16 & Ponto de Acesso: Revista do Instituto de Ciência da Informação da UFBA (PA): 1981-6766; \\
\hline 17 & Revista ACB: Biblioteconomia em Santa Catarina (RACB): 1414-0594; \\
\hline 18 & Revista Brasileira de Biblioteconomia e Documentação (RBBD): 0100-0691 e 1980-6949; \\
\hline 19 & Revista da Escola de Biblioteconomia da UFMG (REBU): 0100-0829; \\
\hline 20 & Revista de Biblioteconomia \& Comunicação (RBC): 0103-0361; \\
\hline 21 & Revista de Biblioteconomia de Brasília (RBB): 0100-7157; \\
\hline 22 & Revista Digital de Biblioteconomia e Ciência da Informação (RDBCI): 1678-765X; \\
\hline 23 & Revista Ibero-Americana de Ciência da Informação (RICI): 1983-5213; \\
\hline 24 & Transinformação (TRA): 0103-3786. \\
\hline
\end{tabular}

Fonte: Dados da pesquisa.

Todos os periódicos desta pesquisa fazem parte da base $\mathrm{ABCDM}$, que foi escolhida justamente por concentrar revista científicas da área da Ciência da Informação. A ABCDM é uma base de dados de referências bibliográficas científicas de Arquivologia, Biblioteconomia, Ciência da Informação, Documentação e Museologia, mantida pela Faculdade de Ciência da Informação da Universidade de Brasília. Pode ser acessada, direta ou indiretamente, por qualquer pessoa que faça parte da amostra desta pesquisa as seguintes revistas.

Fazem parte da base 34 periódicos de áreas correlatas da Ciência da Informação. Entretanto, foram selecionados para esta pesquisa sobre biblioteca no meio digital apenas os periódicos da área de Biblioteconomia.

\subsection{Discussão}

A produção científica é um indicador importante sobre como um determinado assunto é tratado na área e como é desenvolvido pela comunidade científica.

Os estudos que buscam essa análise são importantes, pois apresentam a fundamentação da ideia de ser imprescindível aos pesquisadores estarem atentos para a forma que o conhecimento foi gerado no seu campo de estudo. As principais formas de análises destes estudos são as pesquisas bibliométricas, que elaboram levantamentos sobre estudos publicados anteriormente (SANTOS, 2015, p.5).

Estudos bibliométricos são aqueles que se propõem a analisar os aspectos quantitativos da produção, disseminação e uso da informação registrada com o objetivo de elaborar previsões e apoiar a tomada de decisão, constitui-se de aplicações de técnicas estatísticas e matemáticas para descrever aspectos da literatura (HAYASHI; LETA, 2013). O termo foi mencionado a primeira vez, em 1934, por Otlet

A bibliometria será a parte definida da bibliografia que se ocupa da medida ou da quantidade aplicada ao livro. Todos os elementos considerados pela bibliografia são, em princípio, suscetíveis de mensuração, e deve-se procurar, cada vez mais, exprimir seus dados pela forma precisa do número, em vez de passar ao estado qualitativo ou descritivo ao quantitativo (FONSECA, 1986, p.20-21).

São muitos os indicadores e as variáveis para avaliar a produção científica, variam de acordo com o objetivo e o enfoque definido, entretanto, esses indicadores podem ser organizados em quatro principais categorias: produtividade científica e tecnológica, uso e qualidade dos documentos, redes sociais e colaborativas e relações entre os temas (HAYASHI; LETA, 2013).

As leis bibliométricas são a base para as análises métricas e são: Lei de Bradford, Lei de Lotka, Lei de Zipf (Quadro 2).

Quadro 2 - Leis bibliométricas

\begin{tabular}{|c|c|c|}
\hline Lei & Ano & Descrição \\
\hline Lei de Lotka & 1926 & $\begin{array}{l}\text { Estuda a produtividade dos autores por } \\
\text { meio da identificação e da frequência de } \\
\text { publicações. }\end{array}$ \\
\hline $\begin{array}{l}\text { Lei de } \\
\text { Bradford }\end{array}$ & 1934 & $\begin{array}{l}\text { Relaciona a dispersão da publicação } \\
\text { de artigos de periódicos de um assunto } \\
\text { em revistas destinadas a outros } \\
\text { assuntos e, ainda, auxilia bibliotecários } \\
\text { no desenvolvimento de políticas de } \\
\text { aquisição e descarte de periódicos sobre } \\
\text { determinados assuntos. }\end{array}$ \\
\hline Lei de Zipf & 1949 & $\begin{array}{l}\text { Estuda a frequência do uso de uma } \\
\text { palavra e a sua relação em um } \\
\text { determinado texto. As palavras mais } \\
\text { usadas indicam o assunto do documento. }\end{array}$ \\
\hline
\end{tabular}

Fonte: Noronha e Maricato (2008).

A bibliometria tem caráter interdisciplinar e pode ser aplicada para diferentes áreas do conhecimento para analisar as possibilidades de crescimento de um determinado assunto ou disciplina, identificar autores e instituições que mais produzem, são alguns exemplos de como utilizar essas leis. Nesta pesquisa, o objetivo é analisar o tratamento que a comunidade científica da Ciência da Informação dá aos temas específicos da área, sobretudo, os termos "biblioteca virtual", "biblioteca eletrônica", "biblioteca digital", "biblioteca on-line (online)". 


\subsection{Apresentação e análise dos dados}

Da análise resultou em um conjunto de dados que foram organizados por meio de técnicas de bibliometria e dessa maneira se pode observar os resultados que se seguem:

Quadro 3 - Periódicos científicos e resultados encontrados

\begin{tabular}{|c|c|c|}
\hline ISSN & Periódico Científico & Resultado \\
\hline $0102-4388$ & $\begin{array}{l}\text { Biblos: Revista do Departamento de } \\
\text { Biblioteconomia e História }\end{array}$ & 3 \\
\hline $1981-1640$ & $\begin{array}{l}\text { Brazilian Journal of Information } \\
\text { Science }\end{array}$ & 2 \\
\hline $0102-6607$ & Cadernos de Biblioteconomia & 0 \\
\hline $0007-9421$ & $\begin{array}{l}\text { Cadernos de Biblioteconomia, } \\
\text { Arquivística e Documentação }\end{array}$ & 6 \\
\hline $\begin{array}{l}0100-1965 ; \\
1518-8353\end{array}$ & Ciência da Informação & 44 \\
\hline $1517-3801$ & DatagramaZero & 10 \\
\hline $\begin{array}{l}1807-8893 ; \\
1808-5245\end{array}$ & Em Questão & 3 \\
\hline $1518-2924$ & Encontros Bibli & 11 \\
\hline $2178-2075$ & $\begin{array}{l}\text { InCID: Revista de Ciência da } \\
\text { Informação e Documentação }\end{array}$ & 3 \\
\hline $\begin{array}{l}1414-2139 ; \\
1981-8920\end{array}$ & Informação \& Informação & 3 \\
\hline $\begin{array}{l}0104-0146 ; \\
1809-4783\end{array}$ & Informação \& Sociedade & 12 \\
\hline 0104-9461 & Informare & - \\
\hline $\begin{array}{l}1413-9936 \\
1981-5344\end{array}$ & $\begin{array}{l}\text { Perspectivas em Ciência da } \\
\text { Informação }\end{array}$ & 10 \\
\hline $2236-417 X$ & $\begin{array}{l}\text { Perspectivas em Gestão \& } \\
\text { Conhecimento }\end{array}$ & 0 \\
\hline $1981-6766$ & Ponto de Acesso & 3 \\
\hline 1414-0594 & $\begin{array}{l}\text { Revista ACB: Biblioteconomia em } \\
\text { Santa Catarina }\end{array}$ & 7 \\
\hline $\begin{array}{l}0100-0691 ; \\
1980-6949\end{array}$ & $\begin{array}{l}\text { Revista Brasileira de } \\
\text { Biblioteconomia e Documentação }\end{array}$ & 5 \\
\hline 0100-0829 & $\begin{array}{l}\text { Revista da Escola de } \\
\text { Biblioteconomia da UFMG }\end{array}$ & 0 \\
\hline 0103-0361 & $\begin{array}{l}\text { Revista de Biblioteconomia \& } \\
\text { Comunicação }\end{array}$ & 0 \\
\hline $0100-7157$ & $\begin{array}{l}\text { Revista de Biblioteconomia de } \\
\text { Brasília }\end{array}$ & 0 \\
\hline $1678-765 X$ & $\begin{array}{l}\text { Revista Digital de Biblioteconomia e } \\
\text { Ciência da Informação }\end{array}$ & 5 \\
\hline $1983-5213$ & $\begin{array}{l}\text { Revista Ibero-Americana de Ciência } \\
\text { da Informação }\end{array}$ & 2 \\
\hline $0103-3786$ & Transinformação & 11 \\
\hline $2177-3688$ & ENANCIB & 66 \\
\hline \multicolumn{2}{|r|}{ Total } & 214 \\
\hline
\end{tabular}

Fonte: Dados da pesquisa.

Os periódicos Brazilian Journal of Information Science, Cadernos de Biblioteconomia, Perspectivas em Gestão \& Conhecimento, Revista da Escola de Biblioteconomia da UFMG, Revista de Biblioteconomia \& Comunicação e Revista de Biblioteconomia de Brasília foram desconsiderados da análise de dados, porque não apresentaram resultados. O periódico Informare: Cadernos do Programa de PósGraduação em Ciência da Informação também foram desconsiderados por não ser possível filtrar os resultados pelas revistas em seus sites de busca. Quanto ao periódico ENANCIB - Encontro Nacional de Pesquisa em Ciência da Informação, esse foi dividido por motivos de organização. Os outros periódicos foram mantidos e serão analisados.

O gráfico da Figura 1 relaciona a quantidade de publicações por ano, sobre o tema, de cada uma das revistas científicas participantes da amostra.

Figura 1 - Quantidade de publicações dos periódicos

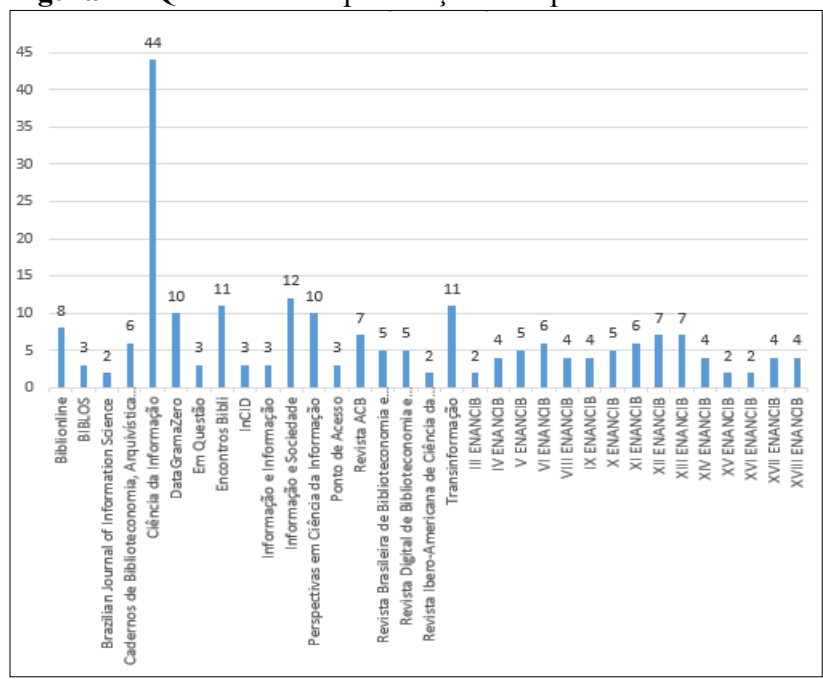

Fonte: Dados da pesquisa.

O periódico que mais publicou foi Ciência da Informação (0100-1965 e 1518-8353;) com 44 artigos, logo em seguida se tem a Revista Informação e Sociedade (0104-0146 e 1809 4783) com 12 publicações, em terceiro lugar está a revista Encontros Bibli: Revista Eletrônica de Biblioteconomia e Ciência da Informação (1518-2924) e Transinformação (0103-3786) com 11 publicações cada.

O gráfico da Figura 2 expõe a análise da quantidade de publicações por ano:

Figura 2 - Quantidades de artigo publicados por ano

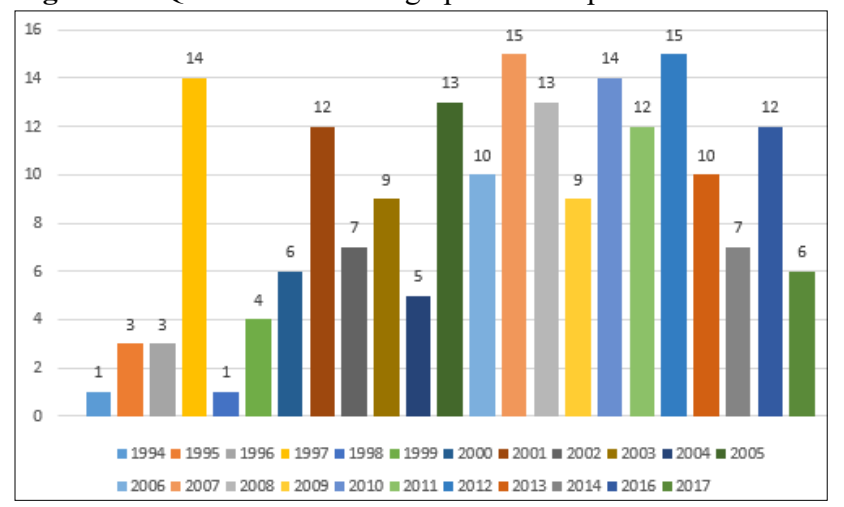

Fonte: Dados da pesquisa.

A partir da observação do Gráfico 2 é possível afirmar que existe uma variedade considerável no número de artigos, que foram publicados entre 1994 e 2017. Verifica-se que 2005 e 2016 foram os anos com produção de uma forma mais constante entre 7 e 13 artigos. O ano de 1997 se destaca com 
14 publicações. Durante todo o restante do período se tem uma variação na produção maior de 1 a 12 artigos por ano. Os anos que tiveram maior publicação foram 1997, 2007, 2010, 2012. É possível notar que nos primeiros três anos - de 1994 até 2003 foram publicados somente 7 artigos.

O gráfico da Figura 3 ilustra a quantidade de autores em cada artigo.

Figura 3 - Quantidade de autores - colaboração

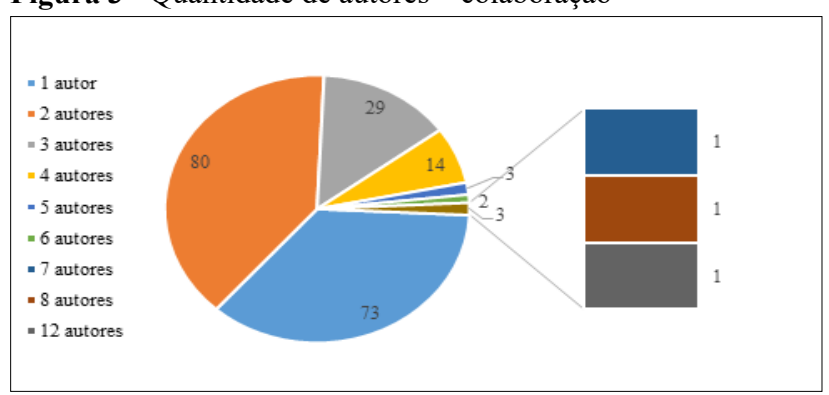

Fonte: Dados da pesquisa.

$\mathrm{Na}$ Figura 3 é possível visualizar essa relação de autoria. Observa-se que 36\% dos artigos possuem um único autor e os outros $39 \%$ dos artigos possuem dois autores, $14 \%$ três autores e outros $11 \%$ possuem autoria múltipla, entre quatro e nove autores em um mesmo artigo.

O gráfico da Figura 4 apresenta os artigos nos quais constavam no título as expressões "biblioteca virtual", "biblioteca eletrônica", "biblioteca digital", "biblioteca online (online)".

Figura 4 - Termos presentes no título

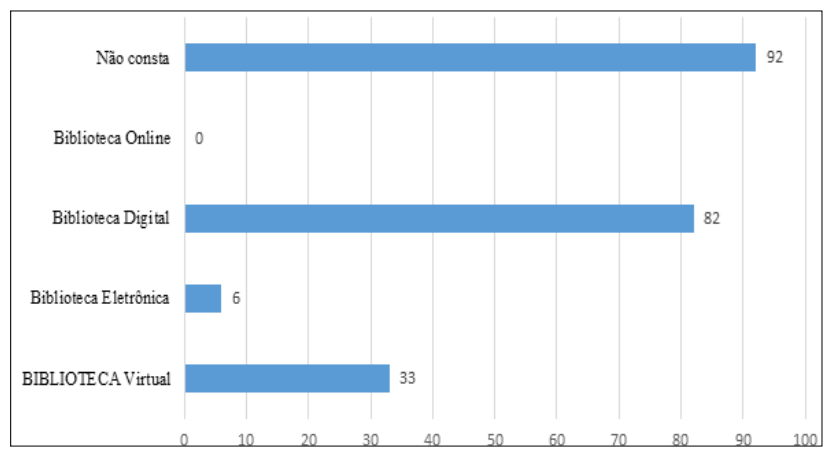

Fonte: Dados da pesquisa.

Observa-se no gráfico da Figura 4 que 92 dos artigos analisados não possuem nenhuma das expressões no título, apenas no campo de palavras-chave, e 121 dos artigos possuem os termos tanto no título quanto no resumo.

\section{Conclusão}

Tendo em vista os dados que foram expostos por meio dos quadros e gráficos, e suas subsequentes análises da amostra dos 19 periódicos usando técnicas básicas de bibliometria, pode-se concluir que é possível identificar os seguintes resultados: quanto à produtividade científica, a Revista Ciência da Informação (0100-1965 e 1518-8353), de acesso aberto, revisada por pares na área de Biblioteconomia e Ciência da Informação com qualis B1 é a que apresenta maior número de publicações.

Quanto à frequência de publicação, o ano que mais se publicou sobre os assuntos foram 2007 e 2012, com 15 artigos cada, aproximadamente uma por mês. Sobre as redes sociais e colaborativas, a maioria dos artigos, $75 \%$ do total, foi escrito por 1 ou 2 autores e o restante possui de 3 a 12 autores. Sobre os que não possuem um dos termos pesquisados no título, $43 \%$ dos artigos, abordam o tema apesar do(s) autor(es) ter(em) preferido usar essa expressão apenas no resumo.

É importante destacar que os termos pesquisados são, de certa forma, considerados sinônimos um dos outros. Por esse motivo foi escolhido especificamente analisar os artigos que possuem no título ou no resumo as expressões "biblioteca virtual", "biblioteca eletrônica", "biblioteca digital”, "biblioteca on-line (online)".

O termo mais utilizado pelos autores que publicaram na base ABCDM para representar o tema foi "biblioteca digital". Não foi recuperado nenhum artigo contendo o termo "biblioteca on-line (online)".

\section{Referências}

FONSECA, E.N. (Coord.). Bibliometria: teoria e prática. São Paulo: Cultrix, 1986.

HAYASHI, M.C.P.I.; LETA, J.(Org.). Bibliometria e cientometria: reflexões teóricas e interfaces. São Carlos: Pedro \& João, 2013.

LAKATOS, E.M.; MARCONI, M.A. Fundamentos de metodologia científica. São Paulo: Atlas, 2010.

NORONHA, D.P.; MARICATO, J.M. Estudos métricos da informação: primeiras aproximações. Enc. Bibli: Rev. Eletr. Bibliotecon. Cienc. Inf., n. esp. p.116-128, 2008. doi: https://doi. org/10.5007/1518-2924.2008v13nesp1p116

SANTOS, G.C. Análise bibliométrica dos artigos publicados como estudos bibliométricos na história do Congresso Brasileiro de Custos. Pensar Contábil, v.17, n.62, p.4-13, 2015. 